$2^{\text {ND }}$ European Conference on Plasma Diagnostics (ECPD2017)

FROM $18^{\text {TH }}$ TO $21^{\text {ST }}$ APRIL 2017

Bordeaux, France

\title{
Conceptual design of a scintillator based Imaging Heavy Ion Beam Probe for the ASDEX Upgrade tokamak
}

\author{
J. Galdon-Quiroga, ${ }^{a, b, 1}$ J.F. Rivero-Rodriguez, ${ }^{b, c}$ M. Garcia-Munoz, ${ }^{a, b}$ G. Birkenmeier, ${ }^{d, e}$ \\ E. Viezzer, ${ }^{a, b}$ J. Ayllon-Guerola, ${ }^{b}$ M. Dunne, ${ }^{d}$ J. Garcia-Lopez, ${ }^{a, b}$ J. Gonzalez-Martin, ${ }^{b, c}$ \\ M.C. Jimenez-Ramos, ${ }^{b}$ M. Rodriguez-Ramos, ${ }^{a, b}$ L. Sanchis-Sanchez, ${ }^{a, b}$ E. Wolfrum ${ }^{d}$ and \\ the ASDEX Upgrade Team ${ }^{d}$ \\ ${ }^{a}$ Department of Atomic, Molecular and Nuclear Physics, University of Seville, 41012 Seville, Spain \\ ${ }^{b}$ Centro Nacional de Aceleradores (CNA), Universidad de Sevilla, \\ Junta de Andalucia, Consejo Superior de Investigaciones Cientificas (CSIC), \\ Parque Cientifico y Tecnologico Cartuja, C/Thomas Alva Edison, 741092 Seville, Spain \\ ${ }^{c}$ ETSI, University of Seville, 41092 Seville, Spain \\ ${ }^{d}$ Max Planck Institute for Plasma Physics, Boltzmannstrasse, 285748 Garching bei Munchen, Germany \\ ${ }^{e}$ Physik-Department E28, Technische Universität München, James-Franck-Str.1, 85748 Garching, Germany \\ E-mail: jgaldon@us.es
}

Abstract: A conceptual design of a new diagnostic for the simultaneous space and time resolved measurement of plasma density, potential and poloidal magnetic field fluctuations at ASDEX Upgrade is proposed. The diagnostic combines the detection techniques of standard heavy ion beam probes (HIBP) and scintillator based fast ion loss detectors (FILD), making use of an atomic beam to probe plasma parameters with high spatio-temporal resolution. This new approach takes advantage of using a neutral probe beam and a scintillator plate as detection system. The combination of these two techniques makes the diagnostic more compact than standard HIBP facilitating its integration in the machine. Simulations using an orbit following code have been carried out to investigate the viability of the proposed detection method based on the displacement of the beam strike-line on the scintillator plate. Relative plasma potential fluctuations from $10 \%$ to $100 \%$ in the potential well induce localized displacements in the strike line in the range of $0.1-1.0 \mathrm{~mm}$, while poloidal magnetic field fluctuations such as those arising from edge currents produce displacements in the order of $\mathrm{mm}$. The use of a scintillator screen provides virtually infinite spatial resolution together with a temporal resolution up to the $\mathrm{MHz}$ range, needed for the identification of internal fluctuations.

Keywords: Plasma diagnostics - probes; Scintillators, scintillation and light emission processes (solid, gas and liquid scintillators)

\footnotetext{
${ }^{1}$ Corresponding author.
} 


\section{Contents}

1 Introduction 1

2 Basics of Imaging Heavy Ion Beam Probe 2

3 Modelling 3

4 Synthetic i-HIBP measurements in ASDEX Upgrade 4

4.1 Proposed experimental setup 4

4.2 Fluctuations in plasma potential and poloidal magnetic field 5

$\begin{array}{lll}4.3 & \text { Fluctuations in plasma density } & 7\end{array}$

5 Conclusions $\quad 8$

\section{Introduction}

Magnetohydrodynamic (MHD) and turbulent fluctuations play a crucial role in the overall performance of magnetically confined fusion plasma devices. A comprehensive experimental characterization of internal plasma fluctuations requires space and time resolved measurements of the fluctuating quantities. Heavy ion beam probes (HIBP) [1-5] are some of the most complete diagnostics for the characterization of small and large scale fluctuations providing simultaneous localized and time resolved measurements of density, potential and poloidal magnetic field perturbations. Indeed, HIBP have been used to investigate a variety of phenomena such as geodesic acoustic mode (GAM) oscillations [6], zonal flows [7, 8], Alfven Eigenmodes (AEs) [9] and radial electric fields at the L-H transition $[10,11]$ among others. More recently, a similar technique was proposed for the study of edge current density [12], which is believed to play a crucial role in the edge localized mode (ELM) stability.

In this paper we propose a new diagnostic concept, the i-HIBP (imaging heavy ion beam probe), that combines the detection technique of a standard HIBP and scintillator based fast ion loss detectors (FILD) [13]. This new concept uses a neutral primary beam and substitutes the secondary beam detection systems used in standard HIBP by a scintillator plate and a fast camera including an appropiate optical system, which makes the system more compact providing an easier implementation in the machine. The detection method relies on the displacement of the strike points of the fan of secondary beams on the scintillator plate and its intensity, providing a virtually infinite spatial resolution of the measurements with high temporal resolution. 
(a)

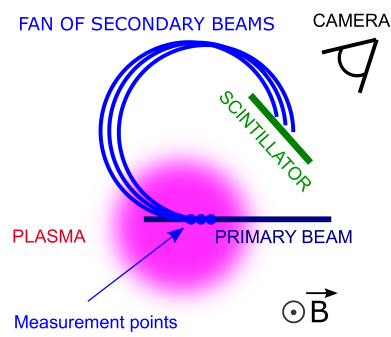

(b)

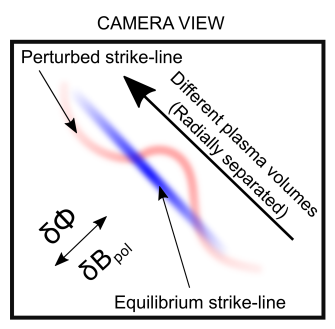

Figure 1. Schematic cartoon showing the measurement principle of an i-HIBP. (a) A probe beam is launched into the plasma. A fan of secondary beams is collected at by a scintillator plate. (b) The strike line at the scintillator provides information at different plasma volumes.

\section{Basics of Imaging Heavy Ion Beam Probe}

The measurement of density and plasma potential fluctuations by means of a standard HIBP [2] relies typically on the use of electrostatic energy analyzers $[1,14,15]$, while for the measurement of poloidal magnetic field fluctuations with better spatial resolution additional systems have been used such as multiple cell array detectors (MCAD) [3, 4]. The new concept proposed here, i-HIBP, substitutes the standard detection systems mentioned above by a scintillator plate and a camera, and uses a neutral primary beam. This measurement technique relies on the imaging of the foot-print of the secondary ions on the scintillator plate, and the displacement of these strike points in the presence of plasma potential and poloidal magnetic field fluctuations. This technique is similar to that used by FILDs. The measurement principle of a i-HIBP is schematically shown in figure 1. A neutral primary beam of heavy atoms is launched into the plasma. The use of a neutral primary beam allows to probe deeper regions of the plasma with relatively low injection energies, achievable for more compact low energy injectors and therefore facilitating considerably the integration in the machine. The beam ionizes along its path and the orbits of the secondary ions are collected with a scintillator. The secondary ions carry information on the plasma potential and density at the volume where the ionization took place, and poloidal magnetic field along the path of the ionized particle. The fan of secondary ions impinging on the scintillator lead to a light emission pattern which has the shape of a line (see figure 1(b)). Its length is related to the ionization profile along the primary beam while its width is determined by the size of the beam. Therefore, different points along the strike line correspond to different plasma volumes along the primary beam. This way, simultaneous measurements of plasma parameters with virtually infinite radial resolution are possible.

In the presence of a plasma potential, the secondary beam will be gaining energy on its path from the ionization point to the scintillator. The energy gain $\left(\Delta E=\left(Z_{\mathrm{sec}}-Z_{\mathrm{prim}}\right) \cdot \Phi_{\text {ion }}\right)$ depends on the plasma potential at the ionization position $\left(\Phi_{\text {ion }}\right)$ and on the charge state of the primaries $\left(Z_{\text {prim }}\right)$ and secondaries $\left(Z_{\mathrm{sec}}\right)$. This energy gain provokes a change in the effective Larmor radius of the ion orbits which is translated to a shift of the strike points on the scintillator. In a similar way, in the presence of a poloidal magnetic perturbation the secondary beam is also deflected due to additional Lorentz force components. The intensity of the strike-line on the scintillator is related to the secondary beam birth profile, which is determined by the density profile of the plasma. Density 
fluctuations would then be measured as intensity fluctuations in the corresponding region of the scintillator.

In contrast to electric detection of secondary ions (i.e: Faraday cups, multi-channel plates or direct collection by electrodes), scintillators avoid the problem of electromagnetic pick-up signals, improving significantly the signal-to-noise ratio in present tokamak harsh environments. The signal can be largely amplified by the scintillator yield $\left(\sim 10^{5}\right.$ photons/ion for certain materials $\left.[16,17]\right)$, enabling the measurement of small density perturbations $(<1 \mu \mathrm{A}$ in the scintillator). The fast decay time of certain scintillator materials $(<1 \mu \mathrm{s})$ would provide a temporal resolution in the order of $\mathrm{MHz}$, needed for the identification of fast fluctuation phenomena.

\section{Modelling}

Modelling efforts have been carried out to assess the viability of the i-HIBP concept in the AUG tokamak. The simulation chain can be divided in two parts: the ion orbit computation of the secondary beams and the beam attenuation. A Fast Ion Orbit Simulator (FIOS) has been developed in this framework. The code solves the Lorentz equation for charged particles in the presence of realistic 3D magnetic and electric fields, using the Boris leap-frog integration method [18-20] and a wall collision method based on the efficient ray-polygon intersection algorithm [21]. The orbits of the secondary ions are computed starting at the ionization positions all along the primary beam path. The finite size of the beam is taken into account in the simulations by distributing the markers in several rings around the beam center. The intensity of the spot in the scintillator is computed analytically taking into account the beam attenuation, which depends on the rate coefficients for the relevant reactions. The primary beam attenuation is given by:

$$
n_{p}\left(l_{0}\right)=n_{p}(0) \cdot e^{-\frac{1}{v_{p}}\left[\int_{0}^{l_{0}} n_{e} \cdot \sum_{i}\langle\sigma v\rangle_{i} d l\right]}
$$

where $n_{p}\left(l_{0}\right)$ is the remaining density of primary beam ions in the position $l_{0} ; n_{p}(0)$ is the initial density of primary beam ions; $v_{p}$ is the velocity of the primary beam given by $v_{p}=\sqrt{\left(\frac{2 E_{p}}{m_{p}}\right) ; n_{e}}$ is the electron density, which is a function of the beam path $l$; and $\langle\sigma v\rangle_{i}$ is the rate coefficients of the relevant reaction $i$ to be taken into account. In this sum all the relevant reaction channels should be included, which will depend on the plasma and beam species and energy. At this stage of the conceptual design, only electron impact ionization reactions have been considered for the computation of the beam attenuation. Charge exchange recombination reactions will be included in the future to evaluate its possible impact on the signal levels.

Similarly, the secondary beam birth profile is given by:

$$
\Delta n_{s}\left(l_{0}\right)=\frac{1}{v_{p}} n_{p}\left(l_{0}\right) n_{e}\left(l_{0}\right)\langle\sigma v\rangle_{\mathrm{sec}} \cdot \Delta l
$$

where $\Delta n_{s}\left(l_{0}\right)$ is the density of generated secondary ions in the interval $\Delta l$ around the position $l_{0}$ and $\langle\sigma v\rangle_{\text {sec }}$ is the rate coefficient of the relevant reaction. The attenuation of the secondary beams is calculated analogously to eq. (3.1). 

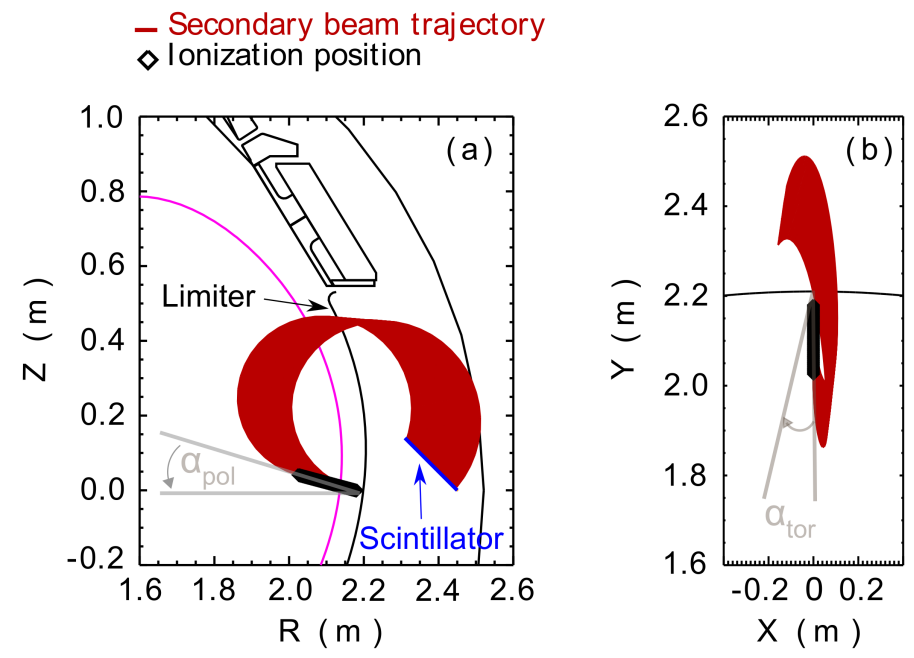

Figure 2. Geometry of the proposed experimental setup at ASDEX Upgrade. The ionization positions of the primary beam are indicated in black. The trajectories of the fan of secondary beams are plotted in red. The injection angles $\alpha_{\mathrm{tor}}$ and $\alpha_{\mathrm{pol}}$ are indicated in grey. (a) Poloidal view. The position of the limiter is highlighted. The scintillator is plotted in blue and the plasma separatrix in magenta. (b) Toroidal view.

\section{Synthetic i-HIBP measurements in ASDEX Upgrade}

Synthetic measurements to estimate the signal level and the sensitivity of the diagnostic to measure fluctuations in the plasma potential, density and edge current have been performed using the FIOS code. In the following, the results of these simulations are presented for a proposed experimental setup at the AUG tokamak, which is first described.

\subsection{Proposed experimental setup}

We propose a conceptual setup for the i-HIBP at the AUG tokamak. The primary beam consists of a neutral ${ }^{133} \mathrm{Cs}$ with an injection energy of $80 \mathrm{keV}$ and a beam diameter of $2 \mathrm{~mm}$ following a Gaussian distribution, with a total intensity of $20 \mu \mathrm{A}$. The use of the low energy neutral beam injection prevents the necessity of a large accelerator. This, in combination with the use of a scintillator plate as detection system, makes the diagnostic more compact than standard HIBP, therefore facilitating the integration in the machine. The detected orbits in the scintillator would be then ${ }^{133} \mathrm{Cs}^{+}$. The beam injection geometry is defined by $\alpha_{\text {tor }}=0^{\circ}$ and $\alpha_{\mathrm{pol}}=15^{\circ}$ launched from $Z=0 \mathrm{~m}$ and $R=2.18 \mathrm{~m}$, where $\alpha_{\text {tor }}$ is the toroidal angle of injection being $0^{\circ}$ the perpendicular (radial) injection and $\alpha_{\mathrm{pol}}$ is the poloidal angle of injection, measured counter-clockwise towards the horizontal plane as indicated in figure 2 .

It should be noticed that in the i-HIBP technique the fan of secondary beams are detected in the scintillator plate before these are able to complete one single gyromotion with a relatively large Larmor radius, therefore leading to a well defined gyrophase of the impinging ions in the scintillator, determined by the primary beam injection geometry. This constitutes a fundamental difference with the FILD diagnostic, in which the fast ions are measured on their orbit after completing many gyromotions with smaller Larmor radius due to the mass difference and therefore leading to a 
randomly distributed gyrophase of the incoming ions. Consequently two main differences can be highlighted between the i-HIBP and the FILD technique. On the one hand, possible contamination of the i-HIBP scintillator signal due to fast ions is unlikely to occur, since the i-HIBP scintillator is located well behind the first wall 3D limiters (see figure 2) and therefore lost ion orbits are prone to collide with any other element of the wall. Additionally, the i-HIBP setup could include a slit which would let in only the ion trajectories corresponding to the fan of secondary beams aimed to be measured. On the other hand, the resolution in energy and pitch angle of the FILD diagnostic is limited due to the fact that the random gyrophase of the incoming ions leads to a dispersion of the strike points in the scintillator, which motivates the inclusion of a 3D collimator to improve the system resolution. On the contrary, in the i-HIBP technique the dispersion of the strike points in the scintillator is only limited by the size of the beam and therefore there is no need to include a collimator. The effect of adding such a collimator would be to effectively reduce the size of the probing beam, which can eventually lead to an improvement of the spatial resolution at a cost of signal level reduction.

Despite the setup proposed here allows measuring all quantitites simultaneously with unprecedented resolution in similar systems, the optimization of the AUG detector geometry is beyond the scope of this work.

We have selected the AUG discharge \#30411 at $5.4 \mathrm{~s}$ as the baseline case for our simulations. A realistic magnetic equilibrium reconstruction was performed using the CLISTE code [22]. In the simulation we also include the radial electric field derived from a typical plasma potential profile at AUG, shown in figure 3 (in black). The $E_{r}$ well is around $-36 \mathrm{kV} / \mathrm{m}$ in this case. The scintillator pattern in absence of the radial electric field is shown in figure 4 (a). Each point in the scintillator pattern corresponds to a different probing volume in the plasma radial coordinate $\rho_{\text {pol }}$. The intensity measured along the strike line corresponds in first order to the birth profile of the beam secondary ions, which is related to the electron density profile (eq. (3.2)). At the bottom right part of the strike line (plasma edge), the primary beam ionizes as it starts exploring regions of increasing electron density. When moving deeper into the plasma, the birth profile of the secondary ions reaches its maximum. From this point on, the attenuation of the primary beam dominates (eq. (3.1)) reducing the production rate of secondary ions and leading to a decrease of the intensity of the strike line towards the top left part (plasma core). The total intensity reaching the scintillator is around $0.2 \mu \mathrm{A}$ and the approximate length of the strike line is $20 \mathrm{~cm}$.

Figure 4(b) shows the difference in the synthetic scintillator signal $\left(\Delta I=I_{w / o E_{r}}-I_{w / E_{r}}\right)$ when the radial electric field is included in the orbit calculations. The strike line in the scintillator is displaced accordingly to the energy gain of the secondary ions following the background potential profile. The absolute displacement of the strike points in the scintillator plane as a function of the probing volume is shown in figure 4(c), revealing a maximum displacement of around $1.2 \mathrm{~mm}$ at the $E_{r}$ well.

\subsection{Fluctuations in plasma potential and poloidal magnetic field}

In order to model the fluctuations in plasma potential several artificial Gaussian-like perturbations have been added to the baseline profile previously used (colored lines in figure 3). Figure 5(a) shows the difference in the scintillator pattern between the baseline case and a relative plasma potential perturbation of $50 \%$ at the potential well $\left(\Delta I=I_{r e f}-I_{\delta \Phi}\right)$. The displacement of the strike line 

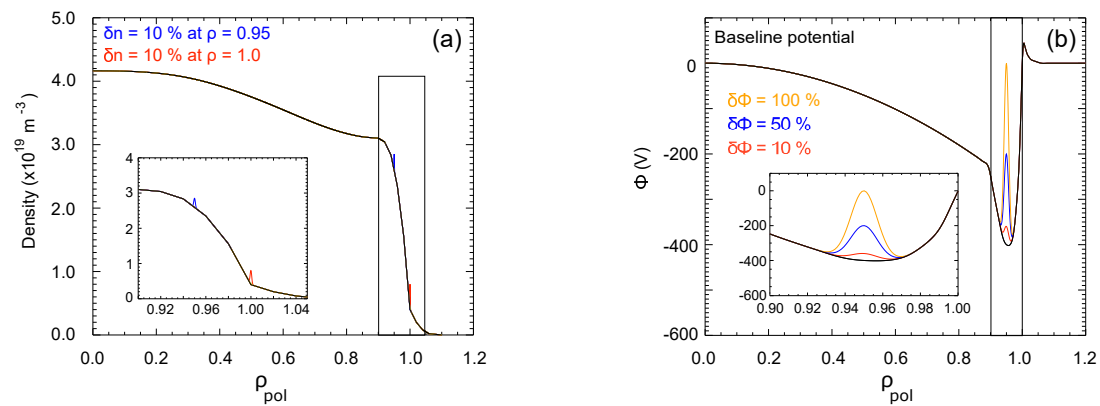

Figure 3. (a) Density profile as a function of $\rho_{\text {pol }}$. In black the equilibrium profile. Gaussian-like perturbations added of $10 \%$ at $\rho_{\text {pol }}=1.0$ (red) and $\rho_{\text {pol }}=0.95$ (blue). (b) Potential profile as a function of $\rho_{\text {pol }}$. In black the equilibrium profile. Gaussian-like perturbations added of $10 \%$ (red), $50 \%$ (blue) and $100 \%$ (yellow).
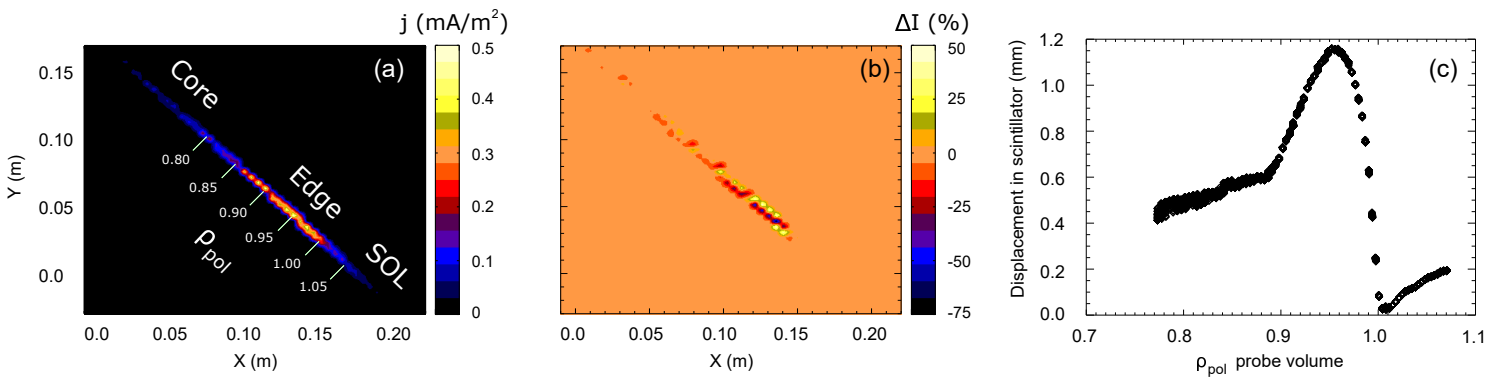

Figure 4. (a) Simulated synthetic scintillator signal in the absence of radial electric field. (b) Difference in the synthetic scintillator signal between the cases with and without radial electric field. (c) Displacement of the strike points in the scintillator as a function of the probing volume. The displacement follows the shape of the plasma potential.
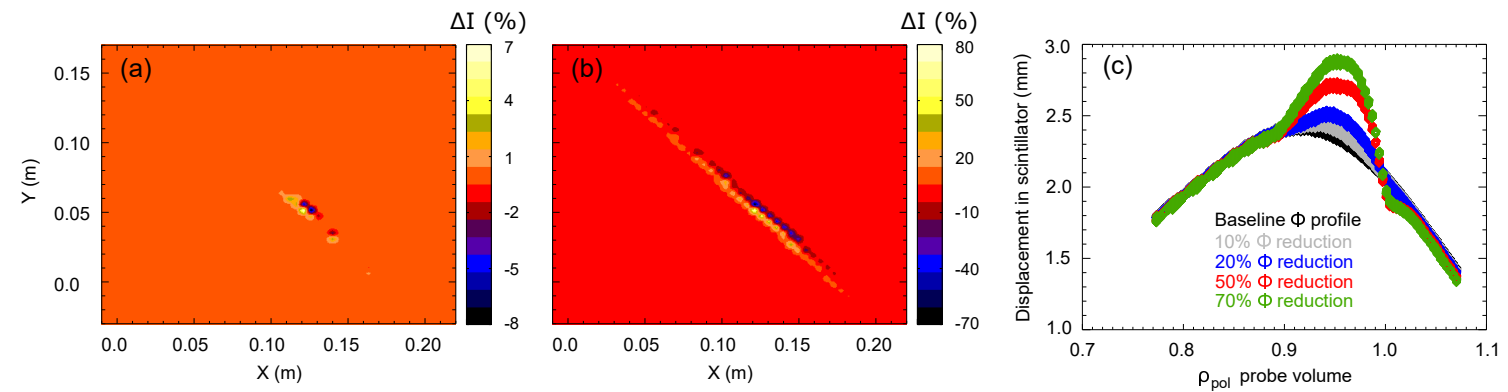

Figure 5. (a) Difference in the synthetic scintillator signal between the baseline case and including a 50\% perturbation in the potential well $(\delta \Phi)$. (b) Difference in the synthetic scintillator signal between the baseline case and including a poloidal magnetic field fluctuation due to an edge current of $5 \mathrm{kA}$. (c) Strike points displacement with respect to the baseline case in the presence of simultaneous edge current fluctuations and several scaled potential profiles. 
is well localized in the position where the perturbation is applied, meaning that the measurements would provide local information on the plasma potential. This result highlights the strength of the iHIBP technique, which should allow for precise determination of the radial position and structure of potential perturbations (such as GAMs or zonal flows). A scan in the amplitude of the perturbation (from $10 \%$ to $100 \%$ ) was carried out, revealing a linear scaling of the displacement of the strike points, ranging from $0.1-1 \mathrm{~mm}$.

Simulations including fluctuations in the poloidal magnetic field, as those arising from the apperance of edge currents, have also been performed. The perturbation was added to the realistic equilibrium current profile. A scan in the intensity of the edge current perturbation was performed using typical values for AUG [23]. Figure 5(b) shows how the strike line in the scintillator is globally displaced ( $\left.\Delta I=I_{\text {ref }}-I_{\delta I_{\text {edge }}}\right)$. In contrast to the plasma potential perturbations, which led to well localized displacements of the strike line, the orbit displacement due to the magnetic field perturbation seems to be subject to integration effects along the ion trajectory. The displacement in this case is in the order of $\mathrm{mm}$ facilitating the detection. A linear scaling is also found between the strike line displacement and the edge current intensity ranging from $0.5-2.5 \mathrm{~mm}$ for currents of $1-5 \mathrm{kA}$.

Possible applications of this diagnostic include the simultaneous measurement of plasma potential and poloidal magnetic field fluctuations. As stated above, the fluctuations in plasma potential induce well localized displacement of the strike line at corresponding position. On the contrary, fluctuations in the poloidal magnetic field induce global displacement of the whole strike line. This behaviour could be used to disentangle both contributions in the presence of simultaneous plasma potential and poloidal magnetic field fluctuations. An example of such an experimental case would be the study of an ELM cycle [24], where the edge current and potential are perturbed due to the reduction of the profile gradients in the pedestal. In order to mimic this situation simulations have been carried out including an edge current fluctuation of $5 \mathrm{kA}$ and reduced potential profiles ( $\Phi_{\text {reduced }}=\Phi_{\text {baseline }} \cdot \lambda$; where $\lambda=\lambda\left(\rho_{\text {pol }}\right)$ is a function such that the potential profile magnitude is reduced in the well but not in the core of the plasma). A scan has been performed in the plasma potential profiles with a reduction of $10 \%, 20 \%, 50 \%$ and $70 \%$ at the well. These correspond to a minimum value of the radial electric field of $-34.4 \mathrm{kV} / \mathrm{m},-29.2 \mathrm{kV} / \mathrm{m},-6.5 \mathrm{kV} / \mathrm{m}$ and $-4.1 \mathrm{kV} / \mathrm{m}$ respectively. These are realistic magnitudes if we compare with typical AUG H-mode plasmas, in which during an ELM crash the edge radial electric field can drop down to typical L-mode like values (minimum of $E_{r}$ smaller than $-10 \mathrm{kV} / \mathrm{m}$ ) [25]. The displacement of the strike points in the scintillator as a function of the probing volume with respect to the baseline case is shown in figure 5(c). The case including only the edge current fluctuation is plotted for reference (black dots). For a small reductions of the potential profile (grey dots) the displacement due to the edge current fluctuation dominates and the different contributions cannot be disentangled. However, if the collapse of the potential profile is large enough (red and green dots) the localized displacement of the strike points can be separated from the global displacement of the strike line due to the edge current fluctuation contribution.

\subsection{Fluctuations in plasma density}

In order to model the fluctuations in the plasma density an artificial perturbation has been added to the baseline density profile (figure 3(a)). The density perturbation is applied to the whole flux surface and therefore it is not poloidally localized. The difference in the intensity measured by the 

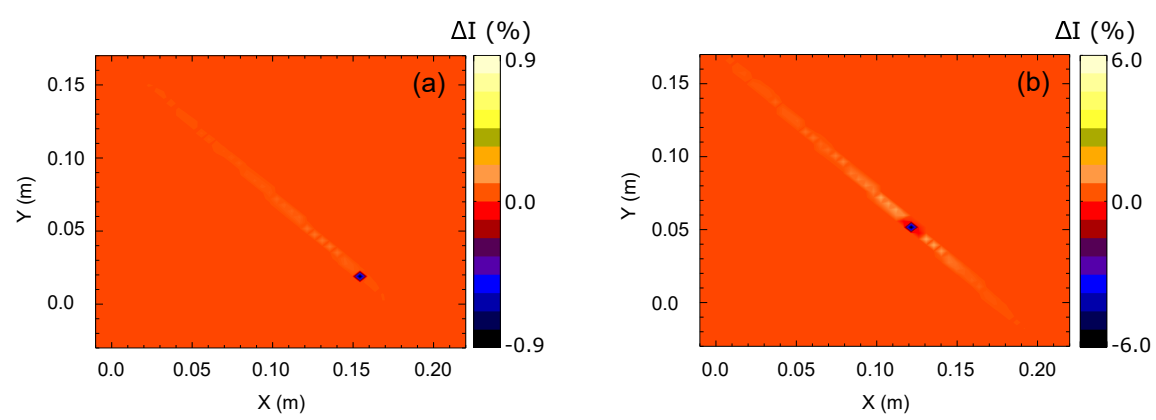

Figure 6. Difference in the synthetic scintillator signal between the baseline case and including a radially localized density perturbation $\left(\delta n_{e}\right)$. (a) $\delta n_{e}=10 \%$ at $\rho_{\mathrm{pol}}=1.0$. (b) $\delta n_{e}=10 \%$ at $\rho_{\mathrm{pol}}=0.95$.

scintillator including a density perturbation of $10 \%$ at $\rho_{\text {pol }}=1.0$ with respect to the baseline case is shown in figure 6(a). The density perturbation leads to a localized increase of the strike line intensity at the location of the perturbation (blue spot) followed by a smaller decrease of the signal intensity along the rest of the strike line (yellow feature). This pattern is due to the relative beam attenuation of the primary beam after exploring the perturbed density region and/or additional attenuation of the secondary beam. Changing the localization of the density perturbation to $\rho_{\text {pol }}=0.95$ reveals that, in fact, the secondary beam attenuation might not be negligible (figure 6(b)). The scintillator pattern shows now an increase in the signal intensity at the location of the density perturbation (in blue), and a decrease in the signal intensity all along the rest of the strike line (in yellow). The decrease in the signal intensity in the region corresponding to $\rho_{\text {pol }}>0.95$ can only be explained by the fact that the non-poloidally-localized density fluctuations are picked-up by the secondary beams on their path out of the plasma. These path effects suggest that modelling of the primary and secondary beam attenuation would be needed for a correct interpretation of the experimental signal [26].

\section{Conclusions}

The concept for a new atomic beam probe diagnostic dedicated to the simultaneous measurement of plasma potential, density and poloidal magnetic field fluctuations has been presented. The proposed concept, i-HIBP, substitutes the detection systems typically used in the standard HIBP by a scintillator plate and uses a neutral primary beam from a dedicated low energy injector. The latter combination makes the system more compact and facilitates the integration in the machine while the use of a scintillator plate as detection system allows the simultaneous measurement of plasma parameters with unprecedented spatial resolution. Simulations including ion orbit calculations and beam attenuation show the effect of different perturbations in the measurement. The length of the strike line in the scintillator is in the order of $20 \mathrm{~cm}$ and measurements in the radial region $\rho_{\text {pol }}>0.8$ seem to be possible for the considered AUG setup. Despite path integration effects due to primary beam attenuation, the intensity pattern of the strike line could be used for density profile reconstruction similarly to what is routinely done for beam emission spectroscopy by means of collisional modelling [27]. Displacements of the strike points in the scintillator in the order of $\mathrm{mm}$ are expected in the presence of edge current perturbations as they tipically appear in inter-ELM 
phases. Displacements in the range of $0.1-1 \mathrm{~mm}$ are expected in the presence of plasma potential perturbations. The fact that the latter induces localized displacement of the strike points indicates that the simultaneous potential and poloidal magnetic field fluctuations, as those occurring during an ELM cycle, may be disentangled.

\section{Acknowledgments}

This research was supported in part by the Spanish Ministry of Economy and Competitiveness (Grant Nos. RYC-2011-09152, FIS2015-69362-P, and ENE2012- 31087) and the Marie Curie FP7 Integration Grant (No. PCIG11-GA-2012-321455).

\section{References}

[1] P. Schoch et al., Heavy-ion beam probe diagnostic systems, Rev. Sci. Instrum. 59 (1988) 1646.

[2] T. Crowley et al., Rensselaer heavy ion beam probe diagnostic methods and techniques, IEEE Trans. Plasma Sci. 22 (1994) 291.

[3] J. Cabral et al., The heavy ion beam diagnostic for the tokamak ISTTOK, IEEE Trans. Plasma Sci. 22 (1994) 350.

[4] I.S. Bondarenko et al., Installation of an advanced heavy ion beam diagnostic on the TJ-II stellarator, Rev. Sci. Instrum. 72 (2001) 583.

[5] A. Melnikov et al., Control and data acquisition for dual HIBP diagnostics in the TJ-II stellarator, Fusion Eng. Des. 96-97 (2015) 724.

[6] A. Melnikov et al., Investigation of geodesic acoustic mode oscillations in the T-10 tokamak, Plasma Phys. Control. F. 48 (2006) S87.

[7] P. Schoch et al., Zonal flow measurements using a heavy ion beam probe, Rev. Sci. Instrum. 74 (2003) 1846.

[8] Y. Hamada et al., Zonal flows in the geodesic acoustic mode frequency range in the JIPP T-IIU tokamak plasmas, Nucl. Fusion 45 (2005) 81.

[9] A. Melnikov et al., Internal measurements of Alfvén eigenmodes with heavy ion beam probing in toroidal plasmas, Nucl. Fusion 50 (2010) 084023.

[10] T. Ido et al., Observation of the Fast Potential Change at L-H Transition by a Heavy-Ion-Beam Probe on JFT-2M, Phys. Rev. Lett. 88 (2002) 055006.

[11] T.Kobayashi et al., Spatiotemporal Structures of Edge Limit-Cycle Oscillation before L-to-H Transition in the JFT-2M Tokamak, Phys. Rev. Lett. 111 (2013) 035002.

[12] M. Berta et al., Development of atomic beam probe for tokamaks, Fus. Eng. Des. 88 (2013) 2875.

[13] M. Garcia-Munoz et al., Scintillator based detector for fast-ion losses induced by magnetohydrodynamic instabilities in the ASDEX upgrade tokamak, Rev. Sci. Instrum. 80 (2009) 053503.

[14] K. Connor et al., Advances in heavy-ion beam probing, Rev. Sci. Instrum. 59 (1988) 1673.

[15] T. Ido et al., $6 \mathrm{MeV}$ heavy ion beam probe on the Large Helical Device, Rev. Sci. Instrum. 77 (2006) $10 \mathrm{~F} 523$. 
[16] M. Jimenez-Ramos et al., Characterization of scintillator materials for fast-ion loss detectors in nuclear fusion reactors, Nucl. Instrum. Meth. B 332 (2014) 216.

[17] M. Rodriguez-Ramos, First absolute measurements of fast-ion losses in the ASDEX Upgrade tokamak, talk given at $21^{\text {st }}$ Topical Conference on High Temperature Plasma Diagnostics, Madison, WI, 2016.

[18] E. Hirvijoki et al., ASCOT: Solving the kinetic equation of minority particle species in tokamak plasmas, Comput. Phys. Commun. 185 (2014) 1310.

[19] P. Stoltz et al., Efficiency of a Boris-like integration scheme with spatial stepping, Phys. Rev. ST Accel. Beams 5 (2002) 094001.

[20] G. Penn, P.H. Stoltz, J.R. Cary and J. Wurtele, Boris push with spatial stepping, J. Phys. G 29 (2003) 1719.

[21] D. Badouel et al., An efficient ray-polygon intersection, in Graphic Gems, Academic Press (1990), pp. 390-393.

[22] P. McCarthy, An integrated data interpretation system for tokamak discharges, Ph.D. thesis, University College Cork (1992).

[23] M. Dunne, Inter-ELM evolution of the edge current density profile on the ASDEX Upgrade tokamak, Ph.D. thesis, University College Cork (2014).

[24] E.Wolfrum et al., Overview of recent pedestal studies at ASDEX Upgrade, Nucl. Fusion 55 (2015) 053017.

[25] E.Viezzer et al., High-accuracy characterization of the edge radial electric field at ASDEX Upgrade, Nucl. Fusion 53 (2013) 053005.

[26] N.Dnestrovskij et al., Development of heavy ion beam probe diagnostics, IEEE Trans. Plasma Sci. 22 (1994) 310.

[27] R.Fischer et al., Probabilistic lithium beam data analysis, Plasma Phys. Control. F. 50 (2008) 085009. 